\title{
Massive MIMO signal transmission in spatially correlated channel environments
}

\author{
Chan-Sic Park $^{1 *}$ (D), Yong-Suk Byun ${ }^{1}$, Jeong Woo Lee ${ }^{2}$ and Yong-Hwan Lee ${ }^{1}$
}

\begin{abstract}
Employment of massive multi-input multi-output ( $\mathrm{m}-\mathrm{MIMO}$ ) transmission techniques has recently been considered as a key technology for the provision of high capacity to a large number of users. Zero-forcing beamforming (ZFBF) techniques can be employed to maximize the transmission capacity, but they may require high implementation complexity in multi-user m-MIMO transmission environments. In this paper, we consider multi-user m-MIMO signal transmission with flexible complexity in spatially correlated channel environments. We initially set the beam weight for conventional maximum ratio transmission (MRT), which may experience interbeam interference. Then, we adjust the beam weight to remove the interbeam interference, while taking into account the trade-off between the implementation complexity and the performance. To this end, we sequentially adjust the beam weight to remove the interbeam interference in a descending order of interference power. The more interference sources are removed, the closer the performance of proposed scheme approaches to that of ZFBF. The proposed beamforming (BF) technique can provide performance close to that of ZFBF in highly correlated m-MIMO channel environments, while significantly reducing the implementation complexity.
\end{abstract}

Keywords: Massive multi-input multi-output, Zero-forcing beamforming, Maximum ratio transmission, Complexity, Interbeam interference

\section{Introduction}

Wireless data traffic has dramatically been increasing with vitalization of wireless multimedia services in cellular communication systems. As a consequence, mobile data traffic has grown 4000 -fold over the past 10 years and almost 400 million-fold over the past 15 years [1]. To support explosively increasing wireless traffic demand, there have been an extensively large number of ongoing research works including the improvement of spectral efficiency and the increase of bandwidth and network density. Employment of massive multi-input multi-output (m-MIMO) antenna techniques has been considered as one of key techniques for the improvement of spectral efficiency in advanced cellular communication systems. It may also provide high energy efficiency, making it quite feasible for being applied to green communication systems [2-4].

${ }^{*}$ Correspondence: parkcs7@ttl.snu.ac.k

'School of Electrical Engineering and INMC, Seoul National University,

Gwanak-ro, Gwanak P.O. Box 34, 151-600 Seoul, Republic of Korea

Full list of author information is available at the end of the article
M-MIMO techniques can transmit signal with the high degree of freedom, enabling to simultaneously serve a large number of users with high transmission capacity without the increase of transmission resource. Employment of maximum ratio transmission (MRT) or zeroforcing beamforming (ZFBF) technique has been considered for multi-user m-MIMO signal transmission. The MRT technique can be implemented with low implementation complexity, but it may seriously suffer from inter-beam interference, providing capacity much lower than the ZFBF technique [5-7]. On the other hand, the ZFBF technique can remove the inter-beam interference at the expense of very high implementation complexity, making it unfeasible in $\mathrm{m}$-MIMO environments [8]. There exist many precoding or beamforming (BF) schemes for the downlink transmission of multi-user MIMO systems $[9,10]$. In [9], the multi-user MIMO downlink channel is decomposed into multiple parallel independent singleuser channels. In [10], the general design of multi-user MIMO precoding matrices is proposed considering various circumstances such as closely spaced antennas and the arbitrary number of equipped antennas. Since these 
schemes use singular value decomposition (SVD) technique, they suffer from extensively high complexity when they are applied in the m-MIMO environment. The lowcomplexity BF scheme based on QR decomposition was introduced in $[11,12]$, whose implementation complexity is, however, still high if a massive number of antennas are used. In order to reduce the implementation complexity further, the BF scheme based on the iterative QR decomposition (IQRD) was proposed in [13]. However, in case that the multiplexing order is not full, the IQRD technique may require an additional combining process resulting in the increase of implementation complexity. Thus, a BF scheme with an affordable implementation complexity became an critical issue in the area of research and development for multi-user m-MIMO systems, and the demand for designing such a BF scheme is continuously growing.

In this paper, we propose a flexible complexity BF scheme for a multi-user m-MIMO transmission system, which is designed by considering the trade-off between the implementation complexity and the transmission performance. Unless the power of interference sources to other users is equally distributed, we can achieve the desired performance by removing major interference sources instead of all. We consider the use of a beam weight determined by the MRT as an initial beam weight. Then, we adjust the beam weight to remove major interference sources generated by the MRT. For ease of implementation, we sequentially adjust the beam weight to remove the interbeam interference in a descending order of interference power. The larger the number of interbeam interference sources to be removed, the closer performance to that of ZFBF we can get. In practice, $\mathrm{m}$ MIMO antennas may need to be installed in a small space, which may result in the presence of a high correlation in the m-MIMO channel. When the m-MIMO channel experiences high correlation, the proposed technique can provide performance close to that of ZFBF by only removing a small number of strong interference sources.

The rest of this paper is organized as follows. Section 2 describes the system model under consideration. Section 3 describes the proposed multi-user beamforming (BF) technique in spatially correlated m-MIMO channel environments. Section 4 evaluates the performance of the proposed BF technique by computer simulation. Finally, Section 5 concludes the paper.

\section{System model}

We consider the downlink transmission in a multi-user $\mathrm{m}$-MIMO wireless communication system, where a base station (BS) is equipped with $N_{T}$ transmit antennas to simultaneously serve $K$ users having a single receive antenna. Let $\Omega_{K}$ be a set of $K$ users in service. When the BS transmits signal to $K$ users by means of spatial multiplexing, the received signal of user $k \in \Omega_{K}$ can be represented as

$$
y_{k}=\sqrt{\alpha_{k} P_{k}} \mathbf{h}_{k}^{H} \mathbf{w}_{k} s_{k}+\sum_{l=1, l \neq k}^{K} \sqrt{\alpha_{k} P_{l}} \mathbf{h}_{k}^{H} \mathbf{w}_{l} s_{l}+n_{k}
$$

where $\alpha_{k}$ denotes the large-scale fading coefficient from the BS to user $k, \mathbf{h}_{k}$ denotes the $\left(N_{T} \times 1\right)$ channel vector from the BS to user $k, \mathbf{w}_{k}$ denotes the $\left(N_{T} \times 1\right)$ beamweight vector of user $k, s_{k}$ denotes the signal of user $k$ with $E\left\{\left|s_{k}\right|^{2}\right\}=1, P_{k}$ denotes the transmit power assigned to user $k, n_{k}$ denotes the zero-mean complex circularsymmetric additive white Gaussian noise (AWGN) with variance $\sigma_{k}^{2}$ at the receiver of user $k$, and the superscript $H$ denotes the conjugate-transpose of a matrix or a vector.

\section{Proposed multi-user m-MIMO transmission}

Let $\mathbf{F}\left(=\left[\mathbf{f}_{1} \mathbf{f}_{2} \cdots \mathbf{f}_{k} \cdots \mathbf{f}_{K}\right]\right)$ denote the $\left(N_{T} \times K\right)$ precoding matrix, where $\mathbf{f}_{k}$ is the $k$-th column of $\mathbf{F}$. Then, the beam weight of user $k$ is determined by $\mathbf{w}_{k}=\mathbf{f}_{k} /\left\|\mathbf{f}_{k}\right\|$. The precoding matrix of MRT and ZFBF schemes, denoted by $\mathbf{F}^{\mathrm{MRT}}$ and $\mathbf{F}^{\mathrm{ZF}}$, respectively, can be determined by [14]

$$
\mathbf{F}^{\mathrm{MRT}}=\mathbf{H}
$$

and

$$
\mathbf{F}^{\mathrm{ZF}}=\mathbf{H}\left(\mathbf{H}^{H} \mathbf{H}\right)^{-1}
$$

where $\mathbf{H}$ denotes the $\left(N_{T} \times K\right)$ channel gain matrix from the BS to $K$ users and the $k$-th column of $\mathbf{H}$ is equal to $\mathbf{h}_{k}$. It follows that the beam weights of user $k$ based on MRT and ZFBF are obtained by $\mathbf{w}_{k}^{\mathrm{MRT}}=\mathbf{f}_{k}^{\mathrm{MRT}} /\left\|\mathbf{f}_{k}^{\mathrm{MRT}}\right\|$ and $\mathbf{w}_{k}^{\mathrm{ZF}}=\mathbf{f}_{k}^{\mathrm{ZF}} /\left\|\mathbf{f}_{k}^{\mathrm{ZF}}\right\|$, respectively. The MRT technique may suffer from severe inter-beam interference. The ZFBF technique can avoid the inter-beam interference, but it may require high implementation complexity [8], making it impractical in multi-user m-MIMO transmission environments. In practice, the MRT is preferred to the ZFBF mainly due to the robustness to imperfect channel information and low complexity [15]. We aim to design a BF scheme which can control the amount of interference cancelation taking into account the implementation complexity.

\subsection{Proposed multi-user beamforming}

For multi-user signal transmission with affordable complexity, we consider the removal of $N(<K)$ interference sources instead of all $(K-1)$ inter-beam interference sources. We determine the number $N$ by taking into consideration the trade-off between the implementation complexity and the amount of interference (i.e., transmission performance). For a given $N$, we minimize the amount of inter-beam interference caused by user $k$ by removing $N$ largest interference sources. Let $\Psi_{k, N}=$ 
$\left[\psi_{k, 1}, \psi_{k, 2}, \cdots, \psi_{k, N}\right]$ denote the user index vector representing $N$ users interfered by user $k$ in a descending order of interference power, where $\psi_{k, j}\left(\in \Omega_{K}\right)$ is the user index whose interference power from user $k$ is the $j$-th highest. Then, we define $\mathbf{Z}_{k, N}$ by

$$
\mathbf{Z}_{k, N} \equiv\left[k, \Psi_{k, N}\right]=\left[k, \psi_{k, 1}, \psi_{k, 2}, \cdots, \psi_{k, N}\right]
$$

where the $n$-th element of $\mathbf{Z}_{k, N}$ is denoted by $z_{k, N}$.

We generate the beam weight $\overline{\mathbf{w}}_{k}$ of user $k$ which is initialized as MRT beam weight $\mathbf{w}_{k}^{\mathrm{MRT}}$ and then adjusted to make the resultant beam not yield interference to $N$ users belonging to $\Psi_{k, N}$. For this purpose, we define first the weight adjustment vector $\Delta \mathbf{w}_{k}^{\left(z_{k, n}\right)}$ as

$$
\Delta \mathbf{w}_{k}^{\left(z_{k, n}\right)}=\mathbf{h}_{z_{k, n}}-\sum_{i=1, i \neq n}^{N+1}\left(\mathbf{h}_{z_{k, n}}^{H} \mathbf{e}_{k, i}\right)^{H} \mathbf{e}_{z_{k, i}}
$$

where $\Delta \mathbf{w}_{k}^{\left(z_{k, n}\right)}$ is orthogonal to the channel directions of users $\left\{z_{k, i} \in \mathbf{Z}_{k, N}, 2 \leq i \leq N+1\right.$ and $\left.i \neq n\right\}$ and $\left\{\mathbf{e}_{z_{k, i}} ; 1 \leq i \leq N+1, i \neq n\right\}$ are $\left(N_{T} \times 1\right)$ orthonormal vectors recursively obtained by means of GramSchmidt process, as summarized in Table 1. It is clear from the property of Gram-Schmidt process that $\Delta \mathbf{w}_{k}^{\left(z_{k, n}\right)}$ is orthogonal to $\mathbf{h}_{z_{k, i}}$ for all $z_{k, i} \in \mathbf{Z}_{k, N}$ and $i \neq n$, but not to $\mathbf{h}_{z_{k, n}}$. It may be desirable to adjust further $\Delta \mathbf{w}_{k}^{\left(z_{k, n}\right)}$ to be orthogonal to $\mathbf{h}_{z_{k, n}}$ as well. By letting

$$
\beta_{k}^{\left(z_{k, n}\right)}=-\frac{\mathbf{h}_{z_{k, n}}^{H} \mathbf{w}_{k}^{\mathrm{MRT}}}{\mathbf{h}_{z_{k, n}}^{H} \Delta \mathbf{w}_{k}^{\left(z_{k, n}\right)}},
$$

we can make $\mathbf{h}_{z_{k, n}}^{H}\left(\mathbf{w}_{k}^{\mathrm{MRT}}+\beta_{k}^{\left(z_{k, n}\right)} \Delta \mathbf{w}_{k}^{\left(z_{k, n}\right)}\right)=0$ for all $n$ and $\mathbf{h}_{z_{k, n}}^{H}\left(\mathbf{w}_{k}^{\mathrm{MRT}}+\sum_{i=2}^{N+1} \beta_{k}^{\left(z_{k, i}\right)} \Delta \mathbf{w}_{k}^{\left(z_{k, i}\right)}\right)=0$ for any $n \neq 1$ because $\mathbf{w}_{k}^{\mathrm{MRT}}=\mathbf{h}_{k}$ and $\mathbf{h}_{z_{k, n}}^{H} \Delta \mathbf{w}_{k}^{\left(z_{k, i}\right)}=0$ for $n \neq i$. It is clear from $\mathbf{h}_{k}=\mathbf{h}_{z_{k, 1}}$ and the property of $\mathbf{h}_{z_{k, 1}}^{H} \Delta \mathbf{w}_{k}^{\left(z_{k, i}\right)}=0$ for all $i \neq 1$ that

$$
\begin{aligned}
\mathbf{h}_{k}^{H}\left(\mathbf{w}_{k}^{\mathrm{MRT}}+\sum_{i=2}^{N+1} \beta_{k}^{\left(z_{k, i}\right)} \Delta \mathbf{w}_{k}^{\left(z_{k, i}\right)}\right) & =\mathbf{h}_{k}^{H} \mathbf{w}_{k}^{\mathrm{MRT}} \\
& =\left\|\mathbf{h}_{k}\right\| .
\end{aligned}
$$

Table 1 Computation of $\mathbf{e}_{z_{k, i}}, 1 \leq i \leq N+1, i \neq n$, for a given $z_{k, n}$

$$
\begin{aligned}
& \text { Initialization } \\
& \text { 1: } \mathbf{e}_{z_{k, 1}}=\mathbf{w}_{k}^{M R T} \\
& \text { 2: for } i=2: N+1 \\
& \text { 3: if } i \neq n \\
& \text { 4: } \quad \mathbf{e}_{z_{k, i}}=\frac{\mathbf{h}_{z_{k, j}-} \sum_{j=1, j \neq n}^{i-1}\left(\mathbf{h}_{z_{k, j}}^{H} \mathbf{e}_{z_{k, j}}\right)^{H} \mathbf{e}_{z_{k, j}}}{\mathbf{h}_{z k, i}-\sum_{j=1, j \neq n}^{i-1}\left(\mathbf{h}_{z_{k, j}}^{H} \mathbf{e}_{z_{k, j}}\right)^{H} \mathbf{e}_{z_{k, j}} \|} \\
& \text { 5: end } \\
& \text { 6: end }
\end{aligned}
$$

As a result, we can make the beam of user $k$ not to yield interference to all users in $\Psi_{k, N}$ by determining the beam weight as

$$
\overline{\mathbf{w}}_{k}=\frac{\mathbf{w}_{k}^{\mathrm{MRT}}+\Delta \mathbf{w}_{k}}{\left\|\mathbf{w}_{k}^{\mathrm{MRT}}+\Delta \mathbf{w}_{k}\right\|}
$$

where

$$
\Delta \mathbf{w}_{k}=\sum_{i=2}^{N+1} \beta_{k}^{\left(z_{k, i}\right)} \Delta \mathbf{w}_{k}^{\left(z_{k, i}\right)} .
$$

Note that $\mathbf{h}_{k}$ and $\Delta \mathbf{w}_{k}$ are orthogonal to each other, i.e., $\mathbf{h}_{k}^{H} \Delta \mathbf{w}_{k}=0$. For each $k \in \Omega_{K}$, we repeat the above process to obtain the corresponding beam weight $\overline{\mathbf{w}}_{k}$.

\subsection{Performance analysis of multi-user beamforming}

We evaluate the performance of BF schemes in terms of the average spectral efficiency defined by

$$
E\left\{C_{k}\right\}=E\left\{\log _{2}\left(1+\gamma_{k}\right)\right\}
$$

where $\gamma_{k}$ is the instantaneous received signal-tointerference-plus-noise ratio (SINR) of user $k$ defined by

$$
\gamma_{k}=\frac{\alpha_{k}\left|\mathbf{h}_{k}^{H} \mathbf{w}_{k}\right|^{2} P_{k}}{\sum_{l=1, l \neq k}^{K} \alpha_{k}\left|\mathbf{h}_{k}^{H} \mathbf{w}_{l}\right|^{2} P_{l}+\sigma_{k}^{2}} .
$$

Let $\lambda_{k}\left(=\alpha_{k} P_{k} / \sigma_{k}^{2}\right)$ be the signal-to-noise ratio (SNR) of user $k$ including the large-scale fading effect. We assume that all users have the same SNR, i.e., $\lambda_{k}=\lambda$.

The average spectral efficiency of user $k$ with beam weight $\overline{\mathbf{w}}_{k}$ obtained by (8) can be expanded as

$$
\begin{aligned}
& E\left\{C_{k}^{\text {prop }}\right\}=E\left\{\log _{2}\left(1+\frac{\alpha_{k}\left|\mathbf{h}_{k}^{H} \overline{\mathbf{w}}_{k}\right|^{2} P_{k}}{\sum_{l=1, l \neq k}^{K} \alpha_{k}\left|\mathbf{h}_{k}^{H} \overline{\mathbf{w}}_{l}\right|^{2} P_{l}+\sigma_{k}^{2}}\right)\right\} \\
& =E\left\{\log _{2}\left(1+\frac{\lambda\left|\mathbf{h}_{k}^{H} \frac{\left(\mathbf{w}_{k}^{\mathrm{MRT}}+\Delta \mathbf{w}_{k}\right)}{\left\|\mathbf{w}_{k}^{\mathrm{MRT}}+\Delta \mathbf{w}_{k}\right\|}\right|^{2}}{\lambda \sum_{l \notin \Psi_{k, N}}\left|\mathbf{h}_{k}^{H} \frac{\left(\mathbf{w}_{l}^{\mathrm{MRT}}+\Delta \mathbf{w}_{l}\right)}{\left\|\mathbf{w}_{l}^{\mathrm{MRT}}+\Delta \mathbf{w}_{l}\right\|}\right|^{2}+1}\right)\right\} .
\end{aligned}
$$

As $N$ increases, the average spectral efficiency also increases. When $N=0$, the proposed BF becomes the MRT, whose average spectral efficiency is lower-bounded by $\log _{2}\left(1+\frac{\lambda\left(N_{T}+1\right)}{\lambda(K-1)+1}\right)$ [14]. When $N=K-1$, the proposed BF provides the same performance as the ZFBF. Note that the MIMO transmission without interference is equivalent to the multi-input single-output (MISO) transmission. It is known that a MISO system with ZFBF can achive an array gain of $\left(N_{T}-K+1\right)$ [16]. It can be shown that the average spectral efficiency of ZFBF is upperbounded by $\log _{2}\left(1+\lambda\left(N_{T}-K+1\right)\right)$. Thus, it can be 
shown that the average spectral efficiency of the proposed $\mathrm{BF}$ is bounded as

$$
\begin{aligned}
& \log _{2}\left(1+\frac{\lambda\left(N_{T}+1\right)}{\lambda(K-1)+1}\right) \leq E\left\{C_{k}^{\text {prop }}\right\} \leq \\
& \log _{2}\left(1+\lambda\left(N_{T}-K+1\right)\right)
\end{aligned}
$$

It can also be shown from $E\left\{\log _{2}(1+x)\right\} \geq$ $\log _{2}\left(1+\frac{1}{E\left\{x^{-1}\right\}}\right)$ that $E\left\{C_{k}^{\text {prop }}\right\}$ is lower-bounded as

$$
\begin{aligned}
E\left\{C_{k}^{\text {prop }}\right\} & \geq \log _{2}\left(1+\left[E\left\{\frac{\sum_{l \notin \Psi_{k, N}}\left|\mathbf{h}_{k}^{H} \overline{\mathbf{w}}_{l}\right|^{2}+\frac{1}{\lambda}}{\left|\mathbf{h}_{k}^{H} \overline{\mathbf{w}}_{k}\right|^{2}}\right\}\right]^{-1}\right) \\
& =\log _{2}\left(1+\left[E\left\{\frac{\sum_{l \notin \Psi_{k, N}}\left|\mathbf{h}_{k}^{H}\left(\tilde{\mathbf{w}}_{l}+\Delta \tilde{\mathbf{w}}_{l}\right)\right|^{2}+\frac{1}{\lambda}}{\left|\mathbf{h}_{k}^{H}\left(\tilde{\mathbf{w}}_{k}+\Delta \tilde{\mathbf{w}}_{k}\right)\right|^{2}}\right\}\right]^{-1}\right)^{(14)}
\end{aligned}
$$

where $\tilde{\mathbf{w}}_{l}=\frac{\mathbf{w}_{l}^{\mathrm{MRT}}}{\left\|\mathbf{w}_{l}^{\mathrm{MRT}}+\Delta \mathbf{w}_{l}\right\|}$ and $\Delta \tilde{\mathbf{w}}_{l}=\frac{\Delta \mathbf{w}_{l}}{\left\|\mathbf{w}_{l}^{\mathrm{MRT}}+\Delta \mathbf{w}_{l}\right\|}$. Assume that the entries of $\overline{\mathbf{w}}_{l}$ and $\mathbf{w}_{l}$ are identically distributed, and that the entries of $\tilde{\mathbf{w}}_{k}$ and $\mathbf{w}_{k}^{\text {MRT }}$ are identically distributed. Then, by the law of large numbers, we obtain $\left|\mathbf{h}_{k}^{H} \tilde{\mathbf{w}}_{k}\right|^{2} \approx\left|\mathbf{h}_{k}^{H} \mathbf{w}_{k}^{\text {MRT }}\right|^{2}$. It follows that

$$
\begin{aligned}
\left|\mathbf{h}_{k}^{H}\left(\tilde{\mathbf{w}}_{k}+\Delta \tilde{\mathbf{w}}_{k}\right)\right|^{2}= & \left|\mathbf{h}_{k}^{H} \tilde{\mathbf{w}}_{k}\right|^{2}+\left|\mathbf{h}_{k}^{H} \Delta \tilde{\mathbf{w}}_{k}\right|^{2} \\
& +\operatorname{Re}\left\{\left(\mathbf{h}_{k}^{H} \tilde{\mathbf{w}}_{k}\right)^{H}\left(\mathbf{h}_{k}^{H} \Delta \tilde{\mathbf{w}}_{k}\right)\right\} \approx N_{T}+1
\end{aligned}
$$

since $\left|\mathbf{h}_{k}^{H} \tilde{\mathbf{w}}_{k}\right|^{2} \approx\left|\mathbf{h}_{k}^{H} \mathbf{w}_{k}^{\mathrm{MRT}}\right|^{2} \approx N_{T}+1$ from the law of large numbers and $\mathbf{h}_{k}^{H} \Delta \tilde{\mathbf{w}}_{k}=0$. Then, we obtain

$$
E\left\{\begin{array}{l}
\left.E \frac{\sum_{l \notin \Psi_{k, N}}\left|\mathbf{h}_{k}^{H}\left(\tilde{\mathbf{w}}_{l}+\Delta \tilde{\mathbf{w}}_{l}\right)\right|^{2}+\frac{1}{\lambda}}{\left|\mathbf{h}_{k}^{H}\left(\tilde{\mathbf{w}}_{k}+\Delta \tilde{\mathbf{w}}_{k}\right)\right|^{2}}\right\} \approx \frac{1}{N_{T}+1} \\
\left.E\left\{\sum_{l \notin \Psi_{k, N}}\left|\mathbf{h}_{k}^{H}\left(\tilde{\mathbf{w}}_{l}+\Delta \tilde{\mathbf{w}}_{l}\right)\right|^{2}\right\}+\frac{1}{\lambda}\right) .
\end{array}\right.
$$

Unlike the proposed BF scheme, when randomly chosen $N$ interference sources are removed, we may assume that $\left\{\mathbf{h}_{k}^{H}\left(\tilde{\mathbf{w}}_{l}+\Delta \tilde{\mathbf{w}}_{l}\right)\right\}$ are identically distributed for all $l \notin$ $\Psi_{k, N}$. Then, we obtain

$$
\begin{aligned}
& E\left\{\sum_{l \notin \Psi_{k, N}}\left|\mathbf{h}_{k}^{H}\left(\tilde{\mathbf{w}}_{l}+\Delta \tilde{\mathbf{w}}_{l}\right)\right|^{2}\right\} \leq(K-1-N) \\
& E\left\{\left|\mathbf{h}_{k}^{H}\left(\tilde{\mathbf{w}}_{l}+\Delta \tilde{\mathbf{w}}_{l}\right)\right|^{2}\right\} .
\end{aligned}
$$

When the channel is uncorrelated, it can be seen that

$E\left\{\left|\mathbf{h}_{k}^{H}\left(\tilde{\mathbf{w}}_{l}+\Delta \tilde{\mathbf{w}}_{l}\right)\right|^{2}\right\} \approx E\left\{\left|\mathbf{h}_{k}^{H} \mathbf{w}_{l}\right|^{2}\right\} \approx 1$, for $l \notin \Psi_{k,(18)}$ since $\left|\mathbf{h}_{k}^{H} \mathbf{w}_{l}\right|^{2}=\frac{\left|\mathbf{h}_{k}^{H} \mathbf{h}_{l}\right|^{2}}{\left\|\mathbf{h}_{l}\right\|^{2}} \approx 1$ from the law of large numbers. Thus, we obtain

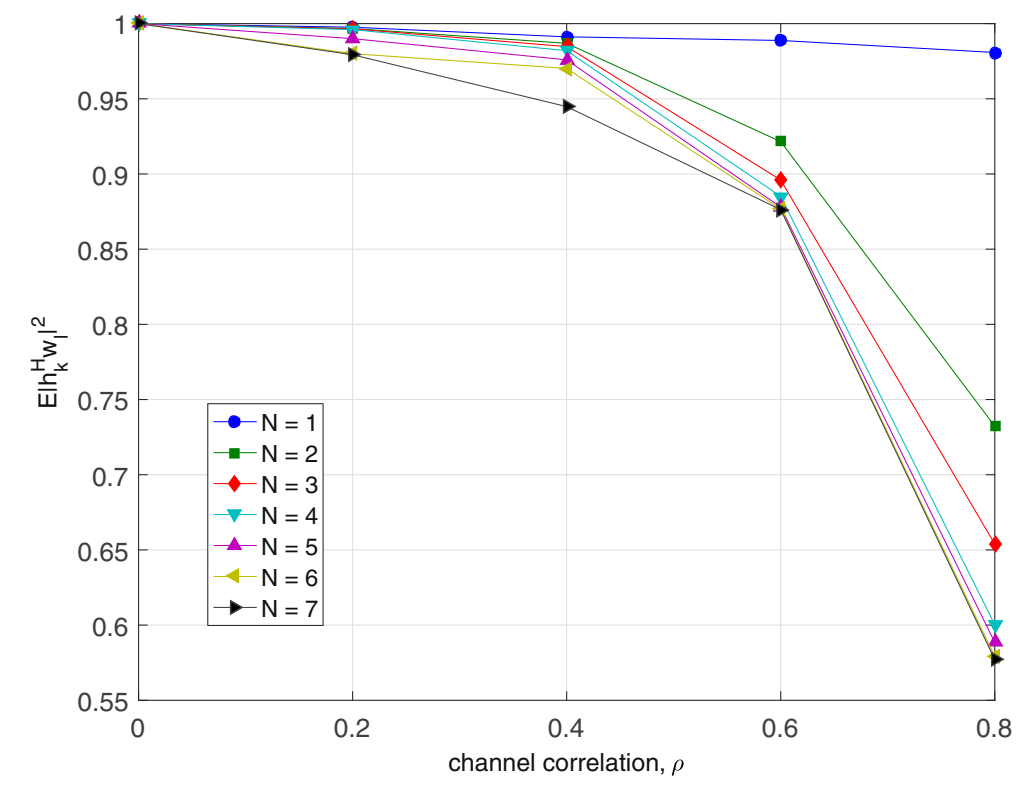

Fig. 1 The value of $E\left\{\left.\left|\mathbf{h}_{k}^{H} \mathbf{w}\right|\right|^{2}\right\}, \mid \notin \boldsymbol{\Psi}_{k, N}$, with respect to $\rho$ for $K=8$ 


$$
E\left\{\sum_{l \notin \Psi_{k, N}}\left|\mathbf{h}_{k}^{H}\left(\tilde{\mathbf{w}}_{l}+\Delta \tilde{\mathbf{w}}_{l}\right)\right|^{2}\right\} \lesssim(K-1-N)
$$

and

$E\left\{\frac{\sum_{l \notin \Psi_{k, N}}\left|\mathbf{h}_{k}^{H}\left(\tilde{\mathbf{w}}_{l}+\Delta \tilde{\mathbf{w}}_{l}\right)\right|^{2}+\frac{1}{\lambda}}{\left|\mathbf{h}_{k}^{H}\left(\tilde{\mathbf{w}}_{k}+\Delta \tilde{\mathbf{w}}_{k}\right)\right|^{2}}\right\} \lesssim \frac{1}{N_{T}+1}\left((K-1-N)+\frac{1}{\lambda}\right)$.

It can also be shown from (14) and (20) that when the channel is uncorrelated, $E\left\{C_{k}^{\text {prop }}\right\}$ is lower-bounded on

$$
E\left\{C_{k}^{\text {prop }}\right\} \gtrsim \log _{2}\left(1+\frac{\lambda\left(N_{T}+1\right)}{\lambda(K-1-N)+1}\right) .
$$

Note that the right-hand side of (21) with $N=0$ equals to the lower-bound of $E\left\{C_{k}^{\text {prop }}\right\}$ given by (13).

Figure 1 depicts $E\left\{\left|\mathbf{h}_{k}^{H} \mathbf{w}_{l}\right|^{2} ; l \notin \Psi_{k, N}\right\}$ with $K=8$ for various channel correlation coefficients $\rho$. It is observed that as $\rho$ grows, $E\left\{\left|\mathbf{h}_{k}^{H} \mathbf{w}_{l}\right|^{2}\right\}$ decreases for all $N$, which implies that the lower bound on $E\left\{C_{k}^{\text {prop }}\right\}$ increases as the channel correlation grows. Note that the decrease of $E\left\{\left|\mathbf{h}_{k}^{H} \mathbf{w}_{l}\right|^{2}\right\}$ results in the decrease of the value of upper bound on $E\left\{\sum_{l \notin \Psi_{k, N}}\left|\mathbf{h}_{k}^{H}\left(\tilde{\mathbf{w}}_{l}+\Delta \tilde{\mathbf{w}}_{l}\right)\right|^{2}\right\}$ as shown in (17) and (18) and the increase of the value of lower bound on $E\left\{C_{k}^{\text {prop }}\right\}$ by (14) and (20).

We measure the implementation complexity of $\mathrm{BF}$ schemes in terms of the number of floating-point operations (FLOPs) [17], which is defined by the total number of multiplications and additions of real numbers. Note that the multiplication and the addition of two complex numbers require six FLOPs and two FLOPs, respectively. For $\left(N_{T} \times 1\right)$ complex vectors $\mathbf{a}$ and $\mathbf{b}$, the inner product $\mathbf{a}^{H} \mathbf{b}$ requires $\left(8 N_{T}-2\right)$ FLOPs, the normalization $\mathbf{a} /\|\mathbf{a}\|$ requires $\left(6 N_{T}-1\right)$ FLOPs, and $\|\mathbf{a}\|$ requires $\left(4 N_{T}-1\right)$ FLOPs.

\begin{tabular}{|c|c|}
\hline Beamforming & FLOP \\
\hline MRT & $K\left(6 N_{T}-1\right)$ \\
\hline ZFBF [13] & $K\left\{\left(24(K-1) N_{T}^{2}+48(K-1)^{2} N_{T}\right)+54(K-1)^{3}\right\}$ \\
\hline \multirow{3}{*}{ IQRD [13] } & $8\left(3 N_{T}-1\right)+\sum_{i=2}^{K-1}(i+1)\left\{8 N_{T}\left[N_{T}-(i-1)\right]\right.$ \\
\hline & $+4\left[3\left(N_{T}-(i-1)\right)-1\right]$ \\
\hline & $\left.+8 N_{T}\left[N_{T}-(i-1)\right]\left(N_{T}-i\right)\right\}$ \\
\hline Proposed BF & $\begin{aligned} K\left[2\left(6 N_{T}-1\right)\right. & +N\left(\left(8 N_{T}-1\right) N^{2}+\left(14 N_{T}-2\right) N\right. \\
& \left.\left.+18 N_{T}+8\right)\right]\end{aligned}$ \\
\hline
\end{tabular}

Table 2 Comparison of the implementation complexity
Let $\Theta^{\mathrm{MRT}}$ and $\Theta^{\beta}$ be the number of FLOPs required to calculate $\mathbf{w}_{k}^{\mathrm{MRT}}$ and $\beta_{k}^{\left(z_{k, i}\right)}$ for each $k$ and $z_{k, i}$, respectively. We also let $\Theta^{\text {gram }}$ be the number of FLOPs required to compute $\Delta \mathbf{w}_{k}^{\left(z_{k, n}\right)}$ for each $z_{k, n}$ and let $\Theta^{\text {norm }}$ be the number of FLOPs for the normalization of an $\left(N_{T} \times 1\right)$ complex vector, where $\Theta^{\text {norm }}=6 N_{T}-1$. It can be shown that the amount of FLOPs required by the proposed BF is

$$
\begin{array}{r}
K\left[\Theta^{\mathrm{MRT}}+N\left(\Theta^{\beta}+\Theta^{\mathrm{gram}}+6 N_{T}\right)+2(N-1) N_{T}\right. \\
\left.+2 N_{T}+\Theta^{\text {norm }}\right]
\end{array}
$$

where $6 N_{T}$ is for the multiplication of $\beta_{k}^{\left(z_{k, i}\right)}$ and $\Delta \mathbf{w}_{k}^{\left(z_{k, i}\right)}$ for each $i$, and $2(N-1) N_{T}$ for the addition of
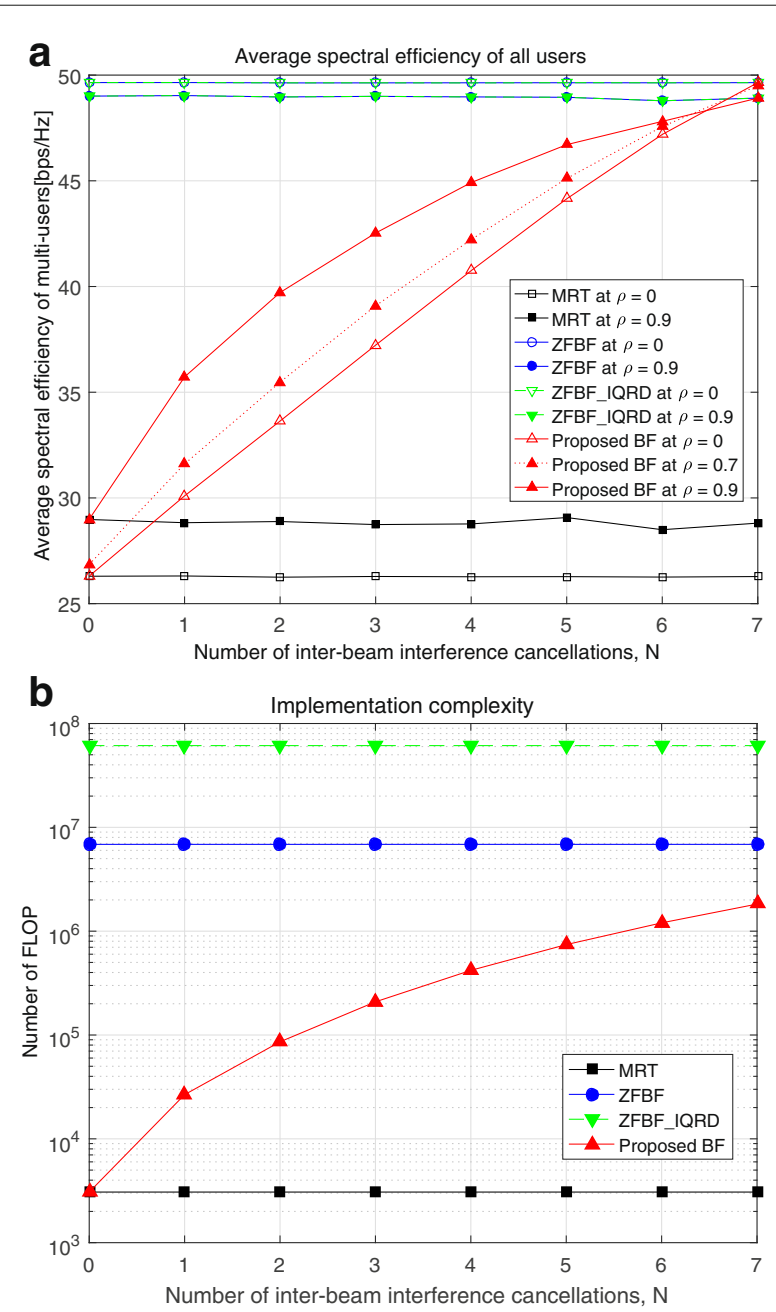

Fig. 2 Average spectral efficiency and implementation complexity with respect to $N$ for $N_{T}=64$. a Average spectral efficiency of all users. b Implementation complexity 
Table 3 The ratio of spectral efficiency and implementation complexity of the proposed BF to those of the ZFBF

\begin{tabular}{|c|c|c|c|c|c|c|c|c|c|}
\hline$N$ & & $0(\%)$ & $1(\%)$ & $2(\%)$ & $3(\%)$ & ) $4(\%)$ & $5(\%)$ & $6(\%)$ & $7(\%)$ \\
\hline \multirow{2}{*}{$\begin{array}{l}\text { Spectral } \\
\text { efficiency }\end{array}$} & $\rho=0.9$ & & 73 & 81 & 87 & 92 & 95 & 99 & 100 \\
\hline & $\rho=0$ & 60 & 73 & 81 & 87 & 92 & 95 & 99 & 100 \\
\hline FLOPs & & 0.04 & 0.38 & 1.25 & 3.04 & 6.11 & 10.82 & 17.53 & 26.59 \\
\hline
\end{tabular}

$\left\{\beta_{k}^{\left(z_{k, i}\right)} \Delta \mathbf{w}_{k}^{\left(z_{k, i}\right)}\right\}$, and $2 N_{T}$ for the addition of $\mathbf{w}_{k}^{\mathrm{MRT}}$ and $\Delta \mathbf{w}_{k}$. It can be shown that $\Theta^{\beta}=2\left(8 N_{T}-2\right)+11=$ $16 N_{T}+7$ and $\Theta^{\mathrm{MRT}}=\Theta^{\text {norm }}=6 N_{T}-1$. As seen in the line 4 of Table 1, we obtain $\left(\mathbf{h}_{z_{k, i}}^{H} \mathbf{e}_{z_{k, j}}\right)^{H} \mathbf{e}_{z_{k, j}}$ for each $j$ using $\left(14 N_{T}-2\right)$ FLOPs, which is repeated $i-1$ times and $i-2$ times if $i<n$ and $i>n$, respectively, to compute $\sum_{j=1, j \neq n}^{i-1}\left(\mathbf{h}_{z_{k, i}}^{H} \mathbf{e}_{z_{k, j}}\right)^{H} \mathbf{e}_{z_{k, j}}$. Afterwards, $\mathbf{h}_{z_{k, i}}$ is added to $\sum_{j=1, j \neq n}^{i-1}\left(\mathbf{h}_{z_{k, i}}^{H} \mathbf{e}_{z_{k, j}}\right)^{H} \mathbf{e}_{z_{k, j}}$ and the normalization is conducted. It can be shown that the Gram-Schmidt process for each $i$ requires $\left[(i-1)\left(16 N_{T}-2\right)+\Theta^{\text {norm }}\right]$ FLOPs for $i<n$, and [ $\left.(i-2)\left(16 N_{T}-2\right)+\Theta^{\text {norm }}\right]$ FLOPs for $i>n$, requiring a complexity of $\Theta^{\text {gram }}$ FLOPs, equal to $\sum_{l=2}^{N+1}\left[(l-1)\left(16 N_{T}-2\right)+\Theta^{\text {norm }}\right]-\Theta^{\text {norm }}$, where the subtraction of $\Theta^{\text {norm }}$ is for the normalization after (5). In a practical multi-user m-MIMO system, $N_{T}$ is much larger than $K$. When $K<N_{T}$, the ZFBF with IQRD additionally requires $K\left\{\left(N_{T}-K+1\right) \Theta^{\text {combine }}+\Theta^{\text {norm }}\right\}$ FLOPs for the generation of precoding vector for each user, where $\Theta^{\text {combine }}=14 N_{T}-2$. For a given $K$, the ZFBF and the proposed BF with $N=K-1$ require implementation complexity of an order of $O\left(N_{T}^{2}\right)$ and $O\left(N_{T}\right)$, respectively. Table 2 summarizes the implementation complexities of $\mathrm{BF}$ schemes under comparison. It is observed that the implementation complexity of the proposed BF is much lower than that of ZFBF.

\section{Performance evaluation}

Consider a multi-user m-MIMO transmission system simultaneously serving $K=8$ users with the number of transmit antennas $N_{T}=32,64,96,128$ at an average SNR of $10 \mathrm{~dB}$. We assume the signal transmission over a channel $\mathbf{H}$ represented as $\mathbf{H}=\mathbf{H}_{0} \mathbf{R}^{1 / 2}$, where the entries of $\mathbf{H}_{0}$ are zero mean independent and identically distributed (i.i.d.) complex Gaussian random variables with unit variance $[18,19]$, and the $(l, m)$-th element of $\mathbf{R}$, denoted by $[\mathbf{R}]_{l, m}$, is represented as $[\mathbf{R}]_{l, m}=\rho e^{j \phi|l-m|}$. Here, $\phi$ is uniformly distributed over $[0,2 \pi)$, unless stated otherwise [20-22]. We evaluate the performance in terms of the average spectral efficiency of all users and the implementation complexity in terms of FLOPs.

In Fig. 2, we depict the average spectral efficiency of all users and the implementation complexity with respect to $N$ for a given $N_{T}=64$. It can be seen from Fig. 2a that as $N$ increases, the performance of the proposed $\mathrm{BF}$ approaches to that of ZFBF. It is observed from Fig. 2a that as $N$ increases, the performance of the proposed $\mathrm{BF}$

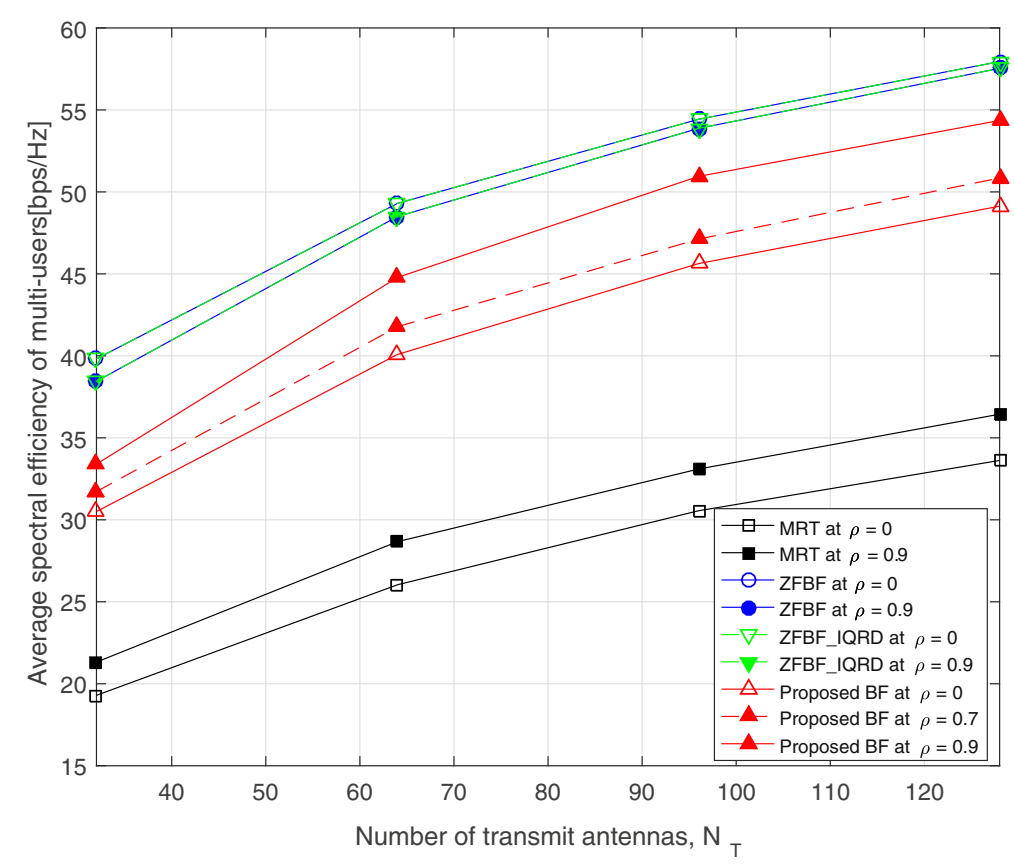

Fig. 3 Average spectral efficiencies of MRT, ZFBF, and the proposed BF with respect to $N_{T}$, where $N=4$ 


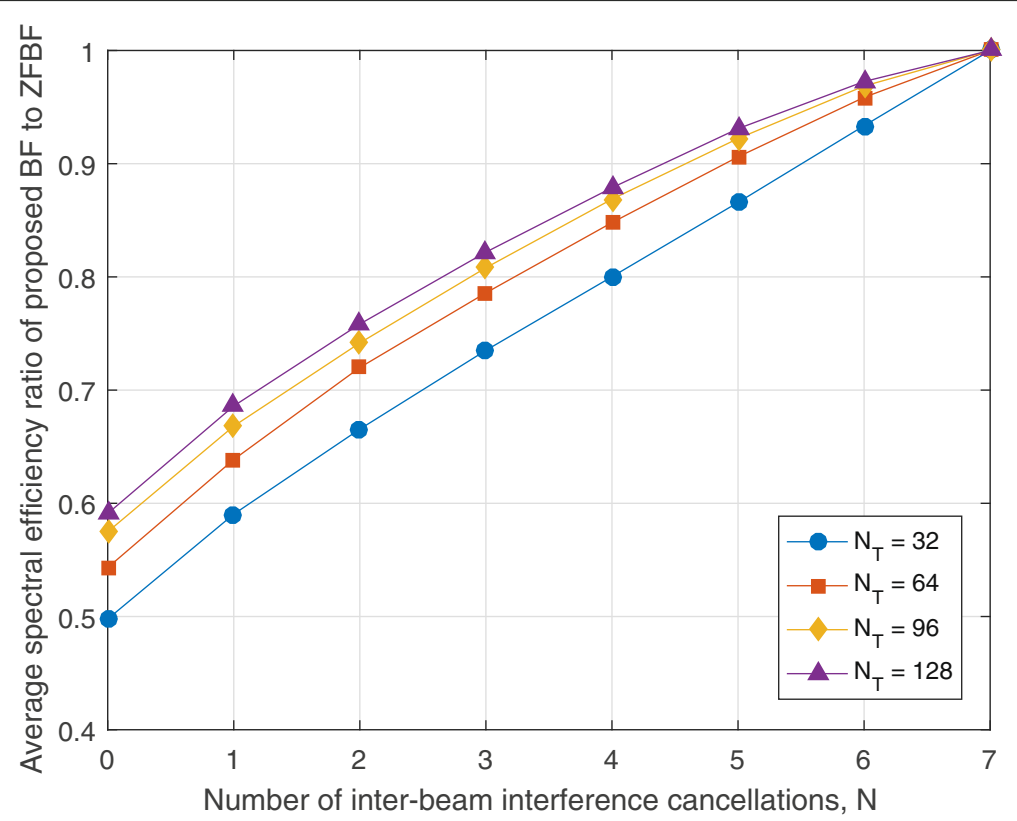

Fig. 4 The ratio of average spectral efficiency of the proposed BF to that of ZFBF with respect to $N$ for some values of $N_{T}$, where $\rho=0.7$

approaches to that of ZFBF. It is also observed that as the spatial correlation increases, the spectral efficiency of ZFBF is somewhat reduced unlike the MRT and the proposed BF increase. Note that as the spatial correlation increases, small singular values of the channel matrix decrease. As a consequence, the singular values of the pseudo-inversed channel matrix corresponding to small singular values of the channel matrix become dominant elements in the ZFBF, yielding the decrease of spectral efficiency [23]. On the other hand, spectral efficiencies of the MRT and the proposed BF increase since the direction of the channel is biased to a singular domain when the channel is highly correlated [23]. It can also be seen that the ZFBF with IQRD requires implementation complexity higher than the ZFBF due to the additional combining process.

Table 3 summarizes the ratio of spectral efficiency and the implementation complexity of the proposed BF to those of the ZFBF according to $N$. It can be seen that when $N=7$, the proposed BF provides the same average

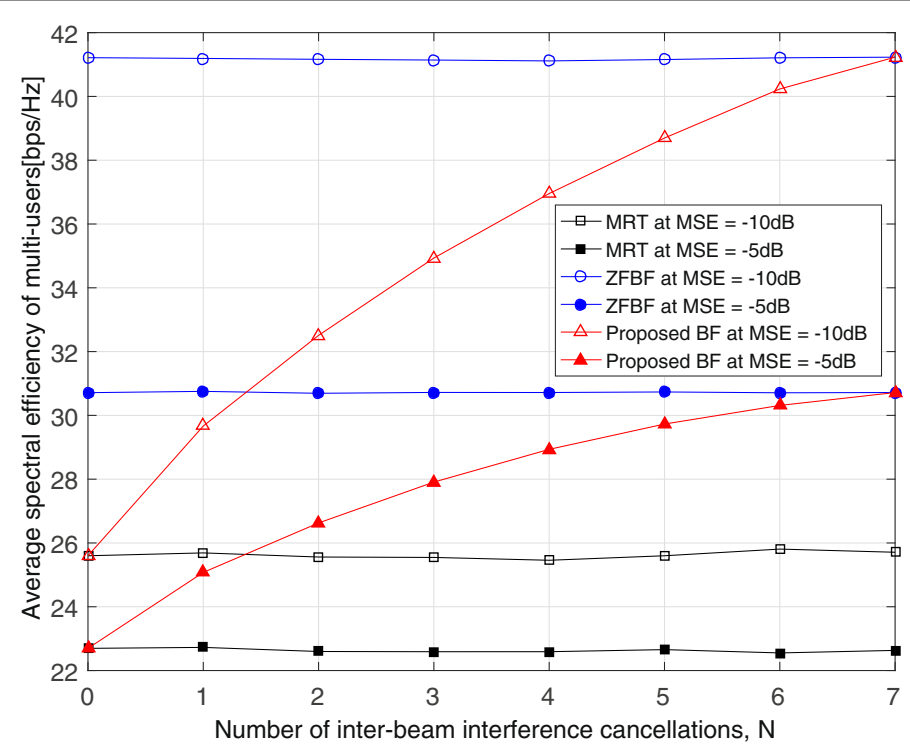

Fig. 5 Average spectral efficiencies of MRT, ZFBF, and the proposed BF in the presence of CSI error, where $\rho=0.7$ is used 
Table 4 The ratio of spectral efficiency obtained by the proposed BF with imperfect CSI to that with perfect CSI, where $N_{T}=64$ and $\rho=0.7$

\begin{tabular}{llllllllll}
\hline$N$ & & $0(\%)$ & $1(\%)$ & $2(\%)$ & $3(\%)$ & $4(\%)$ & $5(\%)$ & $6(\%)$ & $7(\%)$ \\
\hline Spectral efficiency & $\sigma_{\text {mse }}=-10 \mathrm{~dB}$ & 96 & 95 & 93 & 90 & 88 & 86 & 84 & 83 \\
& $\sigma_{\text {mse }}=-5 \mathrm{~dB}$ & 84 & 79 & 75 & 72 & 68 & 66 & 63 & 62 \\
\hline
\end{tabular}

spectral efficiency as the ZFBF, while requiring implementation complexity much lower than the ZFBF. When the channel is highly correlated (e.g., $\rho=0.9$ ), the proposed $\mathrm{BF}$ with $N=4$ can provide an average spectral efficiency higher than $90 \%$ that of ZFBF, while requiring implementation complexity less than $10 \%$ that of ZFBF.

In Fig. 3, we depict the spectral efficiencies of ZFBF, $\mathrm{MRT}$, and the proposed BF with respect to the number of transmit antennas $N_{T}$ obtained for $K=8$ and $N=4$, where various channel correlation coefficients are considered and the average SNR of $10 \mathrm{~dB}$ is used. It is observed that all $\mathrm{BF}$ schemes under comparison achieve higher spectral efficiencies by using higher number of transmit antennas.

In Fig. 4, we plot the ratio of the average spectral efficiency of the proposed BF to that of ZFBF with respect to $N$ for some values of $N_{T}$. It is observed that by using more transmit antennas, the performance of the proposed $\mathrm{BF}$ approaches that of ZFBF more quickly. It follows that in the m-MIMO system with highly correlated channels, the equivalent value of spectral efficiency is achieved by canceling a smaller number of dominant interference sources if we use the higher number of transmit antennas.

Figure 5 depicts the spectral efficiency in the presence of channel estimation error. The estimated channel can be represented as $\hat{\mathbf{w}}_{k}=\left(1-\sigma_{\mathrm{mse}}^{2}\right) \mathbf{h}_{k}+\sigma_{\mathrm{mse}} \sqrt{1-\sigma_{\mathrm{mse}}^{2}} \mathbf{z}_{k}$, where $\sigma_{\text {mse }}$ denotes the mean squared error (MSE) of channel estimation and $\mathbf{z}_{k}$ denotes a $\left(1 \times N_{T}\right)$ random vector whose elements are zero mean complex Gaussian random variables with unit variance [24,25]. It can be seen that as the channel estimation error increases, the performance of all schemes seriously deteriorates. It can also be seen that the transmission performance of the proposed $\mathrm{BF}$ is always between that of the MRT and the ZFBF.

Table 4 summarizes the ratio of spectral efficiency obtained by the proposed BF with imperfect channel information to that with perfect channel information, where $N_{T}=64$ and $\rho=0.7$ are considered. It can be seen that the smaller $N$, the higher robustness to the channel estimation error is attained. It can also be seen that as the channel estimation error increases, the robustness decreases by removing more interference sources.

\section{Conclusions}

We proposed a BF scheme for multi-user m-MIMO signal transmission with flexible implementation complexity. The proposed BF technique can flexibly be implemented by taking into consideration of trade-off between the implementation complexity and the transmission performance, making it quite feasible in real m-MIMO deployment environments.. The numerical results show that the proposed BF is quite effective in the presence of channel correlation, providing transmission performance close to that of ZFBF, while significantly reducing the implementation complexity. The proposed BF technique can be applied to the uplink transmission of the multi-user $\mathrm{m}$-MIMO system in the form of a combiner or a receive $\mathrm{BF}$ scheme implemented at the BS. The beam weight is initialized as a maximal ratio combiner (MRC), and it is adjusted to remove the inter-beam interference in a descending order of uplink interference power considering the tradeoff between the implementation complexity and the performance.

\section{Acknowledgements}

This work was supported by Institute for Information \& communications Technology Promotion(IITP) grant funded by the Korea government(MSIP) (No. R0101-16-244, Development of 5G Mobile Communication Technologies for Hyper-connected Smart Services)

\section{Authors' contributions}

We have considered multi-user beamforming with low implementation complexity in m-MIMO environments. The proposed scheme sequentially cancel out the interbeam interference in an order of the strongest one. Thus, it can compromise the implementation complexity, while maximizing the performance. The numerical and simulation results show that the proposed scheme can significantly reduce the implementation complexity, while providing performance similar to the ZFBF in practical m-MIMO environments.

\section{Competing interests}

The authors declare that they have no competing interests.

\section{Author details}

${ }^{1}$ School of Electrical Engineering and INMC, Seoul National University, Gwanak-ro, Gwanak P.O. Box 34, 151-600 Seoul, Republic of Korea. ${ }^{2}$ School of Electrical and Electronics Engineering, Chung-Ang University, 84 Heukseok-ro, Dongjak-gu, 156-756 Seoul, Republic of Korea.

Received: 26 November 2015 Accepted: 19 September 2016 Published online: 03 October 2016

\section{References}

1. CISCO whitepaper, 2015-2020, (2016)

2. TL Marzetta, in Proc.of Asilomar Conf. on Sign., Syst. and Computers. How much training is required for multiuser MIMO? (2006), pp. 359-363

3. HQ Ngo, EG Larsson, TL Marzetta, Energy and spectral efficiency of very large multiuser MIMO systems. IEEE Trans. Commun. 61(4), 1436-1449 (2013)

4. TL Marzetta, Noncooperative cellular wireless with unlimited numbers of base station antennas. IEEE Trans. Wireless Commun. 9(11), 3590-3600 (2010)

5. Z Shen, et al., Low complexity user selection algorithm for multiuser MIMO systems with block diagonalization. IEEE Trans. Signal Process. 54(9), 3658-3663 (2006) 
6. G Dimic, N Sidiropoulos, On downlink beamforming with greedy user selection: performance analysis and a simple new algorithm. IEEE Trans. Signal Process. 53(10), 3857-3868 (2005)

7. Z Youtuan, T Zhihua, Z Jinkang, in Proc.VTC 2007-Fall. An improved norm-based user selection algorithm for multiuser MIMO systems with block diagonalization, (2007), pp. 601-605

8. Q Spencer, A Swindlehurst, M Haardt, Zero-forcing methods for downlink spatial multiplexing in multiuser MIMO channels. IEEE Trans. Signal Process. 52(2), 461-471 (2004)

9. LChoi, R Murch, A transmit preprocessing technique for multiuser MIMO systems using a decomposition approach. IEEE Trans. Wireless Commun. 3(1), 20-24 (2004)

10. VStankovic, M Haardt, Generalized design of multi-user MIMO precoding matrices. IEEE Trans. Wireless Commun. 7(3), 953-961 (2008)

11. $\mathrm{KZu}, \mathrm{RC}$ de Lamare, Low-complexity lattice reduction-aided regularized block diagonalization for MU-MIMO systems. Commun. Lett. 16(6), 925-928 (2012)

12. $\mathrm{K} Z u, R C$ de Lamare, M Haart, Generalized design of low-complexity block diagonalization type precoding algorithms for multiuser MIMO systems. IEEE Trans. Commun. 61(10), 4232-4242 (2013)

13. L Tran, et al., Iterative precoder design and user scheduling for block-diagonalized systems. IEEE Trans. Signal Process. 60(7), 3726-3739 (2012)

14. CW Lee, et al., Network massive MIMO for cell-boundary users: from a precoding normalization perspective. IEEE Globecom Workshops, 233-237 (2012)

15. H Yang, TL Marzetta, Performance of conjugate and zero-forcing beamforming in large scale antenna systems. IEEE J. Sel. Areas Commun. 31(2), 172-179 (2013)

16. KK Wong, Z Pan, Array gain and diversity order of multiuser MISO antenna systems. Int. J. Wireless Inf. Netw. 2008(15), 82-89 (2008)

17. R Hunger, Floating point operations in matrix-vector calculus, Techneische universitat Munchen associate institute for signal processing, Technical report, (2007)

18. C-N Chuah, D Tse, J Kahn, R Valenzuela, Capacity scaling in MIMO wireless systems under correlated fading. IEEE Trans. Inf. Theory. 48(3), 637-650 (2002)

19. J Kermoal, et al., A stochastic MIMO radio channel model with experimental validation. IEEE J. Sel. Areas Commun. 20(6), 1211-1226 (2002)

20. S Loyka, Channel capacity of MIMO architecture using the exponential correlation matrix. IEEE Commun. Lett. 5(9), 369-371 (2001)

21. C Martin, B Ottersten, Asymptotic eigenvalue distributions and capacity for MIMO channels under correlated fading. IEEE Trans. Wirel. Commun. 3(4), 1350-1359 (2004)

22. K Yu, et al., in Proc.IEEE GLOBECOM. Second order statistics of NLOS indoor MIMO channels based on $5.2 \mathrm{GHz}$ measurements, vol. 1, (2001), pp. $156-160$

23. HCho, et al., Coordinated transmission of interference mitigation and power allocation in two-user two-hop MIMO relay systems. EURASIP J. Wireless Commun. Netw. 2010(2010), 1-15 (2010)

24. TA Thomas, B Mondal, FW Vook, in Proc.IEEE VTC. Method for switching between long term and short term transmit beamforming in OFDM, (2007)

25. AL Garcia, Probability and random processes for electrical engineering (1994)

\section{Submit your manuscript to a SpringerOpen ${ }^{\circ}$ journal and benefit from:}

- Convenient online submission

- Rigorous peer review

- Immediate publication on acceptance

- Open access: articles freely available online

- High visibility within the field

- Retaining the copyright to your article

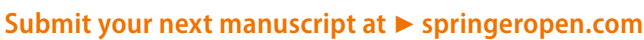

